\title{
Моделирование траектории двикения космического объекта в зоне действия комплексов обнаружения в среде Mathcad
}

\author{
T.В. Калинин ${ }^{1}$, к.т.н., профрессор \\ С.В. Куликов ${ }^{1}$, к.т.н., старший преподаватель \\ Д.В. Хрестинин ${ }^{1}$, преподаватель, diiimaaarik@таil.ru \\ А.С. Кознев ${ }^{1}$, курсант \\ 1 Военно-космическая академия им. А.Ф. Можайского, \\ г. Санкт-Петербург, 197198, Россия
}

При освоении околоземного космического пространства пришлось столкнуться со всевозрастающей опасностью, вызванной ростом количества объектов космического мусора. В силу этого одной из задач радиолокационных и оптико-электронных средств мониторинга околоземного космического пространства является прогнозирование траекторного движения такого рода объектов.

В статье приведены основные положения методики моделирования траектории движения космического объекта в зоне действия наземного радиотехнического и оптико-электронного комплекса космического базирования мониторинга околоземного космического пространства с использованием среды Mathcad. Обоснован достаточно адекватный и в то же время легко внедряемый в реальном масштабе времени способ получения траекторных данных при моделировании околоземной космической обстановки.

Объектом исследования является траектория движения космического мусора искусственного происхождения в зоне действия комплекса обнаружения изменений в обстановке околоземного космического пространства, предметом исследования - модель траектории движения объекта космического мусора искусственного происхождения (техногенного) как составная часть модели обнаружения и сопровождения активными радиотехническими и пассивными оптико-электронными комплексами мониторинга околоземного космического пространства.

Методика моделирования полета космического объекта в зоне действия станции основана на эллиптической теории движения. Параметры зоны действия и технические характеристики радиотехнического комплекса определяются его предназначением.

Методика движения объекта в поле зрения оптико-электронного комплекса, помимо эллиптической теории движения, учитывает технические параметры комплекса, характеристику его движения на околоземной орбите.

Основным результатом исследования являются предложенные методики моделирования, которые позволяют, используя ограниченные вычислительные и графические возможности системы компьютерной математики, достаточно адекватно моделировать траекторию движения космического объекта в зоне действия наземного радиотехнического комплекса с заданными параметрами, а также формировать вектор траекторных измерений в различных системах координат, в том числе и пассивных средств оптико-электронной локации.

Направлениями дальнейшего совершенствования методик являются учет возмущающих факторов, действующих на космический объект при его движении, а также моделирование процессов обработки траекторных измерений маневрирующих космических объектов с различными гипотезами о характере ускорения движения.

Ключевые слова: моделирование, космический объект, радиотехнический комплекс, Mathcad, cuстемы координат, орбита, траектория, оптико-электронный комплекс.

Моделирование процесса обнаружения и сопровождения космических объектов (КО) невозможно без использования модели движения наблюдаемого объекта. Это связано с тем, что, во-первых, изменение во времени параметров отраженного от КО сигнала во многом определяется такими траекторными характери- стиками полета, как наклонная дальность, направление относительно средств наблюдения, скорость, во-вторых, без знания модели движения цели невозможно достоверно имитировать измерения, которые являются входной информацией алгоритмов обнаружения и траекторной обработки радиотехнических (РТК) 
и оптико-электронных комплексов (ОЭК), что и определяет актуальность проведенного исследования.

Моделирование движения КО представляет собой расчет координат его положения и составляющих скорости в заданные моменты времени применительно к выбранной системе координат. Основная сложность моделирования в таком случае заключается в достаточно большом количестве необходимых для использования в расчетах промежуточных систем координат и, следовательно, в трудоемкости математических вычислений.

Задача моделирования движения КО была решена Чепурновым И.А. и Колмаковым И.В. в Военном институте МГТУ имени Н.Э. Баумана, при этом не был рассмотрен случай наблюдения объекта пассивными средствами локации [1]. В Технологическом институте (Индия) А. Тевари в среде Mathlab осуществлено моделирование движения объекта без учета процесса наблюдения его в зоне действия средств локации [2]. В обоих случаях разработка модели требует развитых навыков программирования.

Современные системы компьютерного моделирования, такие как Mathcad, позволяют использовать возможности развитых вычислительных методов без классической процедуры программирования и предоставляют пользователю удобную для работы среду.

Цель - разработка методик моделирования траектории движения КО в зоне действия комплексов обнаружения и их программная реализация в среде компьютерной математики для использования в учебном процессе и прикладных исследованиях возможностей сопровождения траекторий КО радиолокационными и оптико-электронными средствами мониторинга околоземного космического пространства для долговременного прогнозирования космической обстановки, обеспечивающей нормальное функционирование космических систем в области ближнего космоса.

\section{Методика моделирования траектории движения КО в зоне действия наземного РТК}

При моделировании траектории движения КО в зоне действия РТК необходимо использовать не менее четырех систем координат: географическую, геоцентрическую, местную (топоцентрическую) прямоугольную, местную (топоцентрическую) сферическую [3, 4].
Моделирование траектории полета КО сводится к расчету координат его положения и составляющих скорости с учетом уравнений движения. Основными способами вычисления координатных параметров являются интегрирование уравнений движения, разложение в ряд Тейлора и применение формул эллиптической теории [5].

Наиболее экономичным по затратам вычислительного времени является метод имитации полета КО на основе эллиптической теории, основанный на представлении траектории движения в форме эллипса. Выигрыш растет с увеличением временного интервала, на котором рассматривается полет КО.

Кеплерова траектория (орбита) представляет собой эллипс, задаваемый следующими элементами: наклонение плоскости орбиты

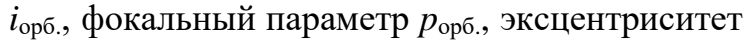

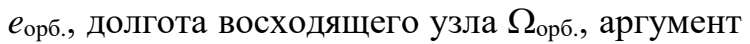

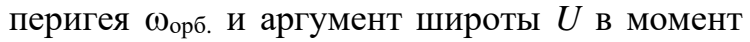
времени $t$. Зная их значения, можно рассчитать

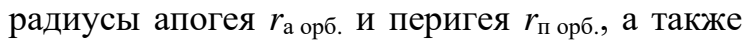
большую полуось орбиты $a_{\text {орб. [1]. }}$

Согласно предлагаемой методике, в системе Mathcad аргумент широты целесообразно выразить через истинную аномалию $v[4,5]$ :

$$
\begin{aligned}
& U(v)=\omega_{\text {орб. }}+v, v_{i i}=i i \cdot t d, \\
& i i=0, \ldots, \frac{360^{\circ}}{t d},
\end{aligned}
$$

где $t d$ - выбранный шаг дискретизации.

Тогда изменение расстояния от центра Земли до КО (радиус орбиты) определяется выражением

$$
r_{0}(v)=\frac{p_{\text {орб. }}}{1+e_{\text {орб. }} \cdot \cos v} .
$$

Скорость движения КО по орбите изменяется по следующему закону:

$$
V(v)=\sqrt{\left(V_{n}(v)\right)^{2}+\left(V_{r}(v)\right)^{2}},
$$

где $V_{n}(\mathrm{v})$ и $V_{r}(\mathrm{v})$ - нормальная и радиальная составляющие скорости соответственно.

Связь координат КО в геочентрической системе координат (ГЦСК) с элементами орбиты задается формулами

$$
\left\{\begin{array}{l}
x_{g}=r_{0}\left(\cos \Omega_{\text {орб. }} \cos U-\sin \Omega_{\text {орб. }} \sin U \cos i_{\text {орб. }}\right), \\
y_{g}=r_{0}\left(\sin \Omega_{\text {орб. }} \cos U+\cos \Omega_{\text {орб. }} \sin U \cos i_{\text {орб. }}\right), \\
z_{g}=r_{0} \sin U \sin i_{\text {орб. }}
\end{array}\right.
$$

В местной прямоугольной системе координат (МПСК) координаты траектории движения КО зависят от заданной в геодезической системе координат (ГСК) точки стояния РЛС 
$\left(\lambda_{0}, \varphi_{0}, h_{30}\right)$ и ее биссектрисы $A z$ :

$$
\left\{\begin{aligned}
x= & x_{g}\left(\sin \lambda_{0} \cos A z-\mathrm{A} \cdot \sin A z\right)- \\
& -y_{g}\left(\cos \lambda_{0} \cos A z+\mathrm{B} \cdot \sin A z\right)+ \\
& +z_{g} \cos \varphi_{0} \sin A z, \\
y= & x_{g} \cdot \mathrm{C}+y_{g} \cdot \mathrm{H}+z_{g} \sin \varphi_{0}-R_{3}-h_{30}, \\
z= & -x_{g}\left(\sin \lambda_{0} \sin A z+\mathrm{A} \cdot \cos A z\right)+ \\
& +y_{g}\left(\cos \lambda_{0} \sin A z-\mathrm{B} \cdot \cos A z\right)+ \\
& +z_{g} \cos \varphi_{0} \cos A z,
\end{aligned}\right.
$$

где $A=\cos \lambda_{0} \sin \varphi_{0}, B=\sin \lambda_{0} \cos \varphi_{0}, C=\cos \lambda_{0}$ $\cos \varphi_{0}, H=\sin \lambda_{0} \cos \varphi_{0}$.

МПСК является промежуточной системой при пересчете координат движения КО из ГЦСК в местную сферическую систему координат (МСфСК). В МСфСК значения угла места $\varepsilon$ и азимута $\alpha$ положения КО относительно РТК зависят от знака координат в МПСК. Это учитывается в программе расчета координат движения КО в МСфСК, созданной в среде Mathcad (рис. 1).

Зона действия РТК в МСфСК определяется заданными параметрами комплекса. Количе- ство таких параметров зависит от выбранного уровня моделирования. Зона действия РТК ограничивается его максимальной $\left(R \_\max \right)$ и минимальной ( $R \_$min) дальностями действия, а также граничными значениями в угломестной ( $\varepsilon \_\min , \varepsilon \_$max) и азимутальной ( $\left.\alpha \_\min , \alpha \_\max \right)$ плоскостях [6]. Приведем программу определения координат движения КО в МСфСК, попадающих в зону действия РТК, реализованную в среде Mathcad (рис. 2).

Данная программа определяет точки траектории КО в МСфСК по всем трем координатам $\left(r_{i}, \varepsilon_{i}, \alpha_{i}\right)$, попадающим в зону действия РТК. Далее координаты этих точек пересчитываются последовательно: вначале в МПСК, а затем в ГЦСК.

В качестве примера применения рассмотренной методики произведено моделирование траектории движения КО с заданными параметрами орбиты: $i_{\text {орб., }} e_{\text {орб., }} \Omega_{\text {орб., }} \omega_{\text {орб., }} p_{\text {орб. }}$ в зоне действия наземного РТК с географическими координатами точки стояния $-\lambda_{0}, \varphi_{0}$. Зона действия модельного РТК, рассчитанная по заданным параметрам РТК и эффективной

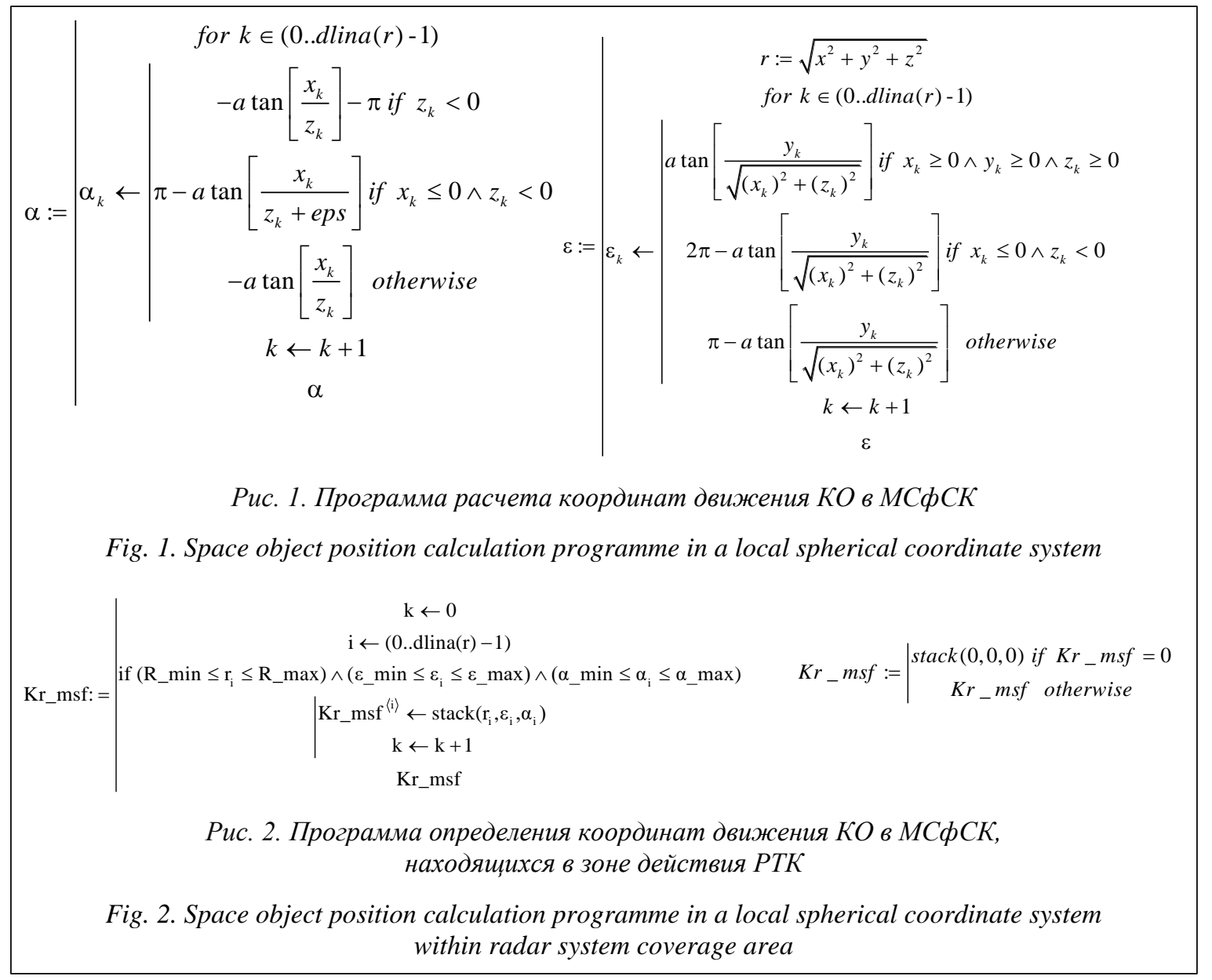


поверхности рассеяния (ЭПР) модельного КО, определяется по максимальной и минимальной дальностям, по углу места и по азимуту.

На рисунке 3 представлена визуализация результатов моделирования с использованием графических возможностей среды Mathcad. Участок траектории движения КО, находящийся в зоне действия РТК, выделен жирной линией.

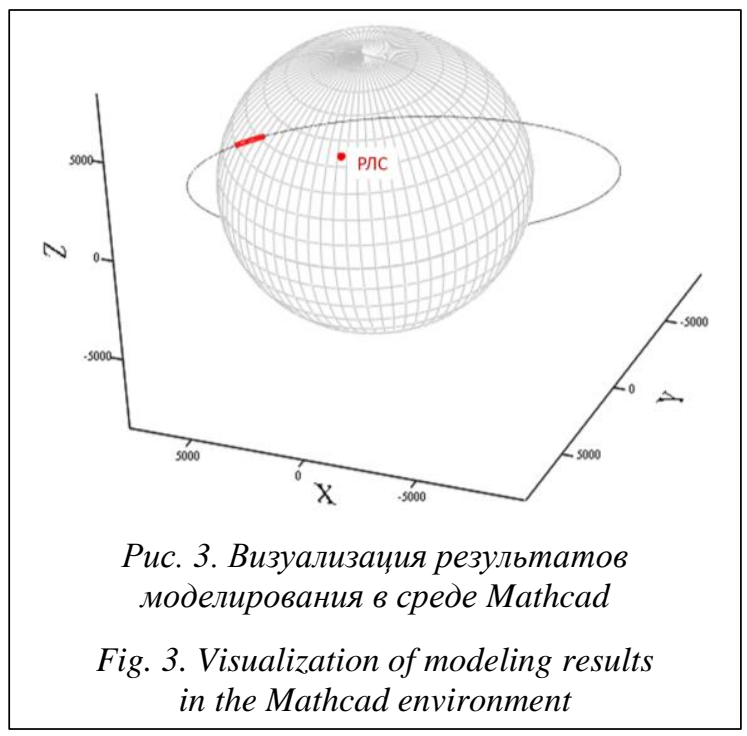

\section{Методика моделирования траектории движения КО в поле зрения ОЭК}

Предположим, что ОЭК, размещенный на борту космического аппарата (КА), включает в себя оптическую систему и фотоприемное устройство (ФПУ). Плоскость ФПУ ортогональна оси оптической системы, совмещенной с осью $X_{1}$ связанной системы координат КА (рис. 4). Дальность до цели не измеряется.

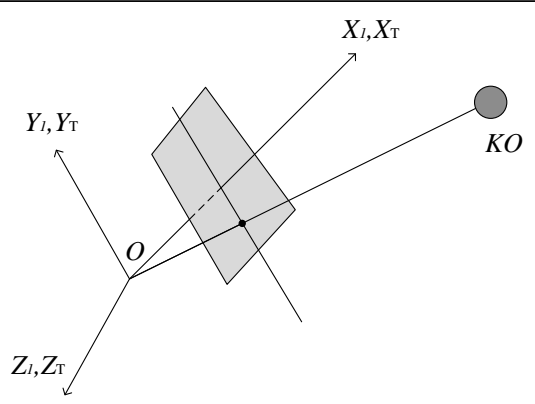

Рис. 4. Ось оптической системы, совмещченная с осью $X_{I}$ связанной системы координат КА

Fig. 4. The axis of the optical system, combined with the $X_{1}$ axis of the associated spacecraft coordinate system
В этом случае в качестве объекта наблюдения в модели динамической системы можно принять изображение цели на ФПУ $[7,8]$.

Соотношения между точками трехмерного пространства и их изображениями на плоскости ФПУ могут быть получены в точке $O$, лежащей на оптической оси и отстоящей от плоскости ФПУ на расстоянии $F$, равном фокусному расстоянию оптической системы.

Допустим, что с ОЭК КА связана приборная система координат (ПрСК) $X_{T} Y_{T} Z_{T}$, начало которой находится в точке $O$ и совпадает с центром масс КА, а оси направлены по связанным осям КА $X_{1} Y_{1} Z_{1}$.

Произведено построение модели динамической системы, включающей модель движения КО, рассмотренную выше, и модель информационной системы. Результаты функционирования системы, полученные в среде Mathcad, приведены на рисунке 5.

\section{Моделирование движения изображения КО на ФПУ ОЭК}

Для моделирования движения изображения КО на плоскости ФПУ требуется найти векторы положения $\rho=\left\|\begin{array}{lll}F & Y_{\Phi} & Z_{\Phi}\end{array}\right\|^{T}$ и скорости $v_{\Phi}=\left\|v_{\Phi Х} v_{\Phi Y} v_{\Phi Z}\right\|^{T}$ изображения КО на ФПУ. Вектор состояния $\left\|\rho_{\Phi}\right\|$ будет определен как вектор состояния точки пересечения линии визирования КО с плоскостью ФПУ [9].

Векторы положения и скорости цели относительно вращающейся с угловой скоростью $\Omega_{\text {КА }}$ системы координат $X_{T} Y_{T} Z_{T}$ запишем в виде

$$
\left\{\begin{array}{l}
\rho=r_{K O}-r=\|X \quad Y \quad Z\|^{T}, \\
v=\left(V_{K O}-V\right)-\Omega_{K A} \cdot \rho=\left\|v_{X} \quad v_{Y} \quad v_{Z}\right\|^{T} .
\end{array}\right.
$$

$$
\text { Векторы }\left\|\begin{array}{l}
r \\
V
\end{array}\right\| \text { и }\left\|\begin{array}{l}
r_{\text {ко }} \\
V_{\text {ко }}
\end{array}\right\| \text { - векторы состояния }
$$

КА и КО, соответственно, относительно инерциального пространства, представленные своими разложениями в системе координат $X_{T} Y_{T} Z_{T}$.

Координаты изображения КО в системе координат ФПУ $Y_{\Phi} Z_{\Phi}$ :

$$
Z_{\Phi}=\frac{Z}{X} F ; Y_{\Phi}=\frac{Y}{X} F \text {. }
$$

Таким образом, радиус-вектор $\rho_{\Phi}$ изображения КО в системе $X_{T} Y_{T} Z_{T}$ равен

$$
\rho_{\Phi}=\frac{F}{X}\|X \quad Y \quad Z\|^{T} .
$$




\begin{tabular}{|c|c|c|c|c|c|c|c|c|c|c|c|}
\hline \multirow{12}{*}{$\operatorname{Data}^{\langle i\rangle}:=$} & \multirow{9}{*}{$\begin{array}{c}\beta_{\mathrm{i}} \\
\mathrm{r}\left(\beta_{\mathrm{i}}\right) \\
\mathrm{v}\left(\beta_{\mathrm{i}}\right) \\
\mathrm{t}\left(\beta_{\mathrm{i}}\right) \\
\frac{\varphi \operatorname{pr}\left(\beta_{\mathrm{i}}\right)}{\operatorname{deg}} \\
\underline{\varphi \operatorname{pr}\left(\beta_{\mathrm{i}}\right)} \\
\end{array}$} & \multirow{8}{*}{ Data:= } & & 0 & 1 & 2 & 3 & 4 & 5 & 6 & 7 \\
\hline & & & 0 & 0 & 0.02 & 0.04 & 0.05 & 0.07 & 0.09 & 0.11 & 0.12 \\
\hline & & & 1 & $6.3 \cdot 10^{6}$ & $6.4 \cdot 10^{6}$ & $6.5 \cdot 10^{6}$ & $6.6 \cdot 10^{6}$ & $6.7 \cdot 10^{6}$ & $6.8 \cdot 10^{6}$ & $6.9 \cdot 10^{6}$ & $7.0 \cdot 10^{6}$ \\
\hline & & & 2 & $7.9 \cdot 10^{3}$ & $7.8 \cdot 10^{3}$ & $7.6 \cdot 10^{3}$ & $7.5 \cdot 10^{3}$ & $7.4 \cdot 10^{3}$ & $7.3 \cdot 10^{3}$ & $7.2 \cdot 10^{3}$ & $7.1 \cdot 10^{3}$ \\
\hline & & & 3 & 0 & 18.88 & 38.33 & 58.36 & 79 & 100.26 & 122.16 & 144.72 \\
\hline & & & 4 & 0 & -1.39 & -2.8 & -4.24 & -5.27 & -7.22 & -8.75 & -10.31 \\
\hline & & & 5 & 50 & 50.5 & 50.98 & 51.44 & 51.88 & 52.31 & 52.72 & 53.1 \\
\hline & & & 6 & 0.06 & 0.05 & 0.05 & 0.04 & 0.04 & 0.04 & 0.04 & 0.03 \\
\hline & & & & & & & & & & & \\
\hline & deg & & & & & & & & & & \\
\hline & $\mathrm{P} 1_{0, \mathrm{i}}$ & & & & & & & & & & \\
\hline & $\mathrm{P} 1_{1, \mathrm{i}}$ & & & & & & & & & & \\
\hline
\end{tabular}

Рис. 5. Результаты моделирования движения КО в среде Mathcad

Fig. 5. The results of motion modeling in the Mathcad environment

Для нахождения вектора скорости изображения КО определим, что вектор угловой скорости линии визирования его в движении относительно вращающейся системы $X_{T} Y_{T} Z_{T}$ равен

$$
\begin{aligned}
& \omega_{\text {ЛВ }}=\frac{\rho v}{\rho^{2}}=\frac{\rho\left(V_{Ц}-V\right)}{\rho^{2}}- \\
& -\Omega_{\text {ЛА }}+\frac{\left(\rho, \Omega_{\text {ЛА }}\right)}{\rho^{2}} \rho=\left\|\begin{array}{l}
\omega_{\text {ЛВ }} \\
\omega_{\text {ЛВ }} \\
\omega_{\text {ЛВZ }}
\end{array}\right\| .
\end{aligned}
$$

Для нахождения вектора скорости изображения КО $v_{\Phi}$ заметим, что Флв может быть выражена через параметры движения изображения КО на плоскости ФПУ:

$$
\omega_{\text {лв }}=\frac{\rho_{\Phi} \times v_{\Phi}}{\rho_{\Phi}^{2}},
$$

и учтем, что проекция скорости $v_{\Phi}$ на ось $X_{T}$ равна нулю, так как плоскость ФПУ ортогональна оси $X_{T}$. Тогда из (10) следует, что

$$
\omega_{\text {лВ } Y}=-F \frac{v_{\Phi Z}}{\rho_{\Phi}^{2}} ; \omega_{л в Z}=-F \frac{v_{\Phi Y}}{\rho_{\Phi}^{2}} .
$$

Отсюда получаем выражение для вектора скорости изображения КО на ФПУ в системе $X_{T} Y_{T} Z_{T}$ :

$$
\begin{aligned}
& v_{\Phi}=\frac{\rho_{\Phi}^{2}}{F}\left\|0 \quad \omega_{\text {ЛВ }} \quad-\omega_{\text {ЛВ }}\right\|^{T}= \\
& =\frac{\rho_{\Phi}^{2}}{F}\left\|\begin{array}{ccc}
0 & 0 & 0 \\
0 & 0 & 1 \\
0 & -1 & 0
\end{array}\right\| \cdot\left\|\omega_{\text {Лв }}\right\| .
\end{aligned}
$$

Полученные соотношения используются для моделирования движения изображения КО на плоскости ФПУ- приборе с зарядовой связью (рис. 6). Векторы состояния КА $\rho_{\Phi}$ и КО $v_{\Phi}$,

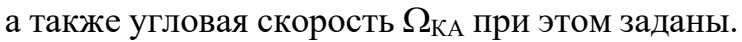

Результаты функционирования модели записываются в два файла с расширениями .txt и .xls, из которых производится считывание данных для визуализации наблюдения траектории движения КО (рис. 6). Данные файлы возможно использовать в качестве исходных данных для других прикладных программных продуктов и систем компьютерной математики, например Mathlab/Simulink [10].

\section{Заключение}

Таким образом, предложенные методики моделирования позволяют, используя вычислительные и графические возможности системы Mathcad, достаточно адекватно моделировать траекторию движения КО в зоне действия наземного РТК с заданными параметрами, а также формировать вектор траекторных измерений в различных системах координат, в том числе и пассивных средств оптикоэлектронной локации.

Направлениями дальнейшего совершенствования методик являются учет возмущающих факторов, действующих на КО при его

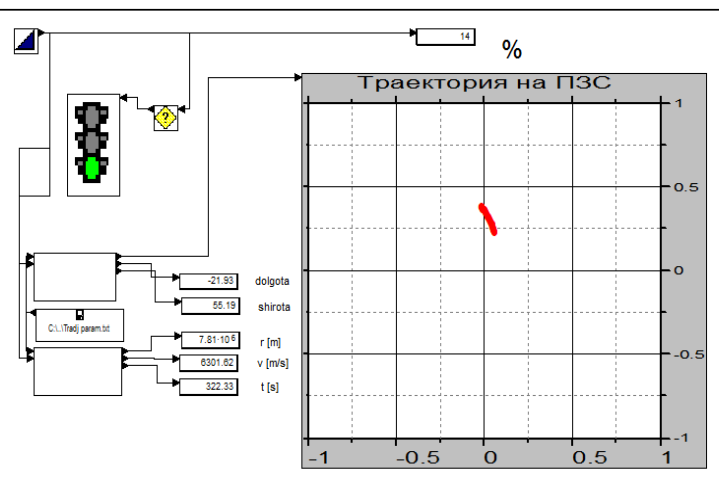

Рис. 6. Моделирование движения изображения иели на плоскости ФПУ

Fig. 6. Image motion modeling on the flat of a photodetector 
движении, а также моделирование процессов обработки траекторных измерений маневриру- ющих объектов с различными гипотезами о характере ускорения движения.

\section{Лumepamypa}

1. Чепурнов И.А., Колмаков И.В. Использование среды Mathlab при моделировании радиолокационных изображений // Актуальные вопросы развития вооружения, военной и специальной техники Войск противовоздушной и противоракетной обороны, Космических войск Воздушно-космических сил: сб. тр. III Всеросс. науч.-практич. конф. 2018. С. 417-421.

2. Ashish Tewari. Advanced Control of Aircraft, Spacecraft and Rockets. John Wiley \& Sons, 2011, 454 p.

3. Васин В.А., Власов И.Б., Егоров Ю.М., Федоров И.Б. [и др.]. Информационные технологии в радиотехнических системах. М.: Изд-во МГТУ им. Н.Э. Баумана, 2003. 672 с.

4. Дьяконов В.П. Mathcad 8-12 для студентов. М.: СОЛОН-Пресс, 2005. 632 с.

5. Чепурнов И.А. Использование современных систем компьютерного моделирования при исследовании сложных систем вооружения и военной техники // Актуальные вопросы развития вооружения, военной и специальной техники Войск противовоздушной и противоракетной обороны, Космических войск Воздушно-космических сил: сб. тр. 2016. С. 276-284.

6. Кротов К.Ю., Шабанов А.А., Чепурнов И.А. Методика оценки характеристик РЛС слежения за космическими объектами // Актуальные вопросы развития вооружения, военной и специальной техники Войск противовоздушной и противоракетной обороны, Космических войск Воздушно-космических сил: сб. тр. 2016. С. 224-233.

7. Чернявский Г.М., Чернов А.А. Лазерные системы в космосе (информационная технология). М.: Радио и связь, 1995. 224 с.

8. Morefield Ch.L. Application of 0-1 integer programing to multitarget tracking problems. IEEE, Transactions on Automatic Control, 2007, vol. 22, no. 3, iss. 3, pp. 302-312. DOI: 10.1109/TAC.1977.1101500.

9. Зотов Р.В., Калинин Т.В. Оптимальная фильтрация траекторной информации. Метод определения параметров движения космических объектов информационными системами пассивной локации // Современные научно-технические проблемы и направления совершенствования вооружения и средств информационного обеспечения войск РКО: сб. матер. 12 науч.-технич. конф. 2007. С. 15-20.

10. Калинин Т.В., Лисицкий В.В., Демидова Н.С., Сервецкий А.И. Математическая модель обнаружения баллистического космического объекта многоспектральным оптико-электронным комплексом // Системы управления, связи и безопасности. 2018. № 3. С. 232-248. URL: http://sccs.intelgr.com/archive/ 2018-03/11-Kalinin.pdf (дата обращения: 20.03.2020).

\section{Trajectory modeling space object in range Mathcad discovery complexes}

T.V. Kalinin ', Ph.D. (Engineering), Professor

S.V. Kulikov ${ }^{1}$, Ph.D. (Engineering), Senior Lecturer

D.V. Khrestinin ${ }^{1}$, Lecturer, diiimaaarik@mail.ru

A.S. Koznev ${ }^{1}$, Cadet

${ }^{1}$ A.F. Mozhaysky Military Space Academy, St. Petersburg, 197082, Russian Federation

Abstract. In the development of near-Earth space had to face an ever-increasing danger caused by the increase in the number of space debris objects. In view of this, one of the problems of radar and optoelectronic means (NES) for monitoring near-Earth space is the prediction of the trajectory movement of such objects.

The paper presents the main provisions of the methodology for modeling the trajectory of a space object in the coverage area of a ground-based radio-technical and optical-electronic complex of space-based monitoring of near-Earth space using the Mathcad medium. The paper substantiates a rather adequate and at the same time easily implemented in real time way to obtain trajectory data in modeling the near-Earth space environment.

The research object is the trajectory of space debris of artificial origin in the area of the complex of detection of changes in the environment of near-Earth space. The study subject is a model of the trajectory of an object of space debris of artificial origin (technogenic) as an integral part of the model of detection and tracking by active radio-technical and passive optoelectronic complexes for monitoring near-Earth space. 
The modeling technique of space object flight in the station coverage area is based on an elliptical theory of motion. The parameters of the coverage area and the technical characteristics of the radio complex are determined by its purpose.

In addition to the elliptical theory of motion, the technique of moving an object in a vision of an opticalelectronic complex takes into account the technical parameters of the complex and the characteristics of its motion in near-Earth orbit.

The main result of the study is the proposed modeling techniques that allow, using the limited computational and graphic capabilities of a computer mathematics system, it is sufficient to adequately simulate the trajectory of a space object in the coverage area of a ground-based radio complex with specified parameters, as well as generate a vector of trajectory measurements in various coordinate systems, in including passive means of the optoelectronic location.

The directions of further improvement of the methods are taking into account disturbing factors acting on the space object during its movement, as well as modeling the processes of processing trajectory measurements of maneuvering space objects with various hypotheses about the nature of the acceleration of motion.

Keywords: modeling, space object, radar system, Mathcad, coordinate systems, orbit, trajectory, optronic system.

\section{References}

1. Chepurnov I.A., Kolmakov I.V. Using Mathlab environment for modeling radar images. Proc. Conf. Major Aspects of the Development of Weapons, Military and Special Equipment of the Air and Anti-Missile Defense, Space Troops of the Aerospace Forces, 2018, pp. 417-421 (in Russ.).

2. Ashish Tewari. Advanced Control of Aircraft, Spacecraft and Rockets. John Wiley \& Sons Publ., 2011, 454 p.

3. Vasin V.A., Vlasov I.B., Egorov Yu.V., Fedorov I.B. (Eds.), et al. Information Technologies in Radio Systems. Moscow, 2003, 672 p. (in Russ.).

4. Dyakonov V.P. Mathcad 8-12 for Students. Moscow, 2005, 632 p. (in Russ.).

5. Chepurnov I.A. Usage of modern computer modeling systems for complex weapon and military equipment systems evaluation. Proc. All-Russ. Sci.-Pract. Conf. Major Aspects of the Development of Weapons, Military and Special Equipment of the Air and Anti-Missile Defense, Space Troops of the Aerospace Forces, 2016, pp. 276-284 (in Russ.).

6. Krotov K.Yu., Shabanov A.A., Chepurnov I.A. Methods for assessing the characteristics of a spacetracking radar system. Proc. All-Russ. Sci.-Pract. Conf. Major Aspects of the Development of Weapons, Military and Special Equipment of the Air and Anti-Missile Defense, Space Troops of the Aerospace Forces, 2016, pp. 224-233 (in Russ.).

7. Chernyavskiy G.M., Chernov A.A. Lazer Systems in Space (Information Technology). Moscow, 1995, 224 p. (in Russ.).

8. Morefield Ch.L. Application of 0-1 integer programing to multitarget tracking problems. IEEE, Transactions on Automatic Control, 2007, vol. 22, no. 3, iss. 3, pp. 302-312. DOI: 10.1109/TAC.1977.1101500.

9. Zotov R.V., Kalinin T.B. Optimal filtering of path information. Method for determining the motion parameters of space objects by passive location information systems. Proc. 12th Sci. Tech. Conf. Modern Scientific-Technical Problems and Directions of Development of Weapons and Missile Ballistic Defense Information Supply, 2007, pp. 15-20 (in Russ.).

10. Kalinin T.V., Lisitskiy V.V., Demidova N.S., Servetsky A.I. Detection model of a ballistic space object with a multi-spectral optical-electronic complex. Systems of Control, Communication and Security, 2018, no. 3, pp. 232-248 (in Russ.). Available at: http://sccs.intelgr.com/archive/2018-03/11-Kalinin.pdf (accessed March 20, 2020).

\section{Для цитирования}

Калинин Т.В., Куликов С.В., Хрестинин Д.В., Кознев А.С. Моделирование траектории движения космического объекта в зоне действия комплексов обнаружения в среде Mathcad // Программные продукты и системы. 2020. Т. 33. № 3. C. 476-482. DOI: 10.15827/0236-235X.131.476482 .

\section{For citation}

Kalinin T.V., Kulikov S.V., Khrestinin D.V., Koznev A.S. Trajectory modeling space object in range Mathcad discovery complexes. Software \& Systems, 2020, vol. 33, no. 3, pp. $476-482$ (in Russ.). DOI: $10.15827 / 0236-235 X .131 .476-482$. 